\title{
DOUBLE MULTIPLIERS ON TOPOLOGICAL ALGEBRAS
}

\author{
L. A. KHAN, N. MOHAMMAD, and A. B. THAHEEM
}

(Received 17 December 1996 and in revised form 13 March 1998)

\begin{abstract}
The main purpose of this paper is to investigate some topological properties of the double multiplier algebra on a topological algebra. Let $M_{d}(A)$ be the double multiplier algebra on a topological algebra $A$, and let $u$ and $s$ be the uniform and strong operator topologies on $M_{d}(A)$, respectively. It is shown, under some additional hypotheses on $A$, that

(1) $M_{d}(A)$ is $u$ - and $s$-complete;

(2) $A$ is a $u$-closed two-sided ideal in $M_{d}(A)$;

(3) $A$ is $s$-dense in $M_{d}(A)$;

(4) $s$ and $u$ have the same bounded sets;

(5) each continuous onto homomorphism $\phi: A \rightarrow B$ has a unique extension $\tilde{\phi}:\left(M_{d}(A)\right.$, $\left.s_{A}\right) \rightarrow\left(M_{d}(B), s_{B}\right)$.
\end{abstract}

Keywords and phrases. Double multipliers, topological algebras, uniform operator topology, strong operator topology.

1991 Mathematics Subject Classification. 46H05, 47C05, 46A16.

1. Introduction. The theory of double multipliers (i.e., of double centralizers) was developed for topological algebras by Johnson [7] and further investigated in the case of Banach algebras and $C^{*}$-algebras by Busby [2], Fontenot [5], Taylor [17], Tomiuk [18], Argün and Rowlands [1], and others; see the monographs [9, 12] for additional references. If $A$ is a commutative $C^{*}$-algebra, that is $A=C_{0}(X)$ - the algebra of all complex-valued continuous functions which vanish at infinity on a locally compact Hausdorff space $X-$, then $M_{d}(A)$, the algebra of all double multipliers of $A$, is $C_{b}(X)$ - the algebra of all complex-valued bounded continuous functions on $X$ [21]. The noncommutative generalization of the relationship between $C_{0}(X)$ and $C(X)$ was found to be useful in the work of Busby [2], Taylor [17], Davenport [4], and Lazar and Taylor [12].

In view of the applications of (nonnormed) topological algebras in other fields such as quantum mechanics and quantum statistics (see, e.g., Lassner $[10,11]$ ) and recent developments in the theory of topological algebras (see, for instance, the book of Mallios [13]), it is important to consider operators on more general classes of topological algebras. More recently, Phillips $[14,15]$ has studied inner and approximately inner derivations on pro- $C^{*}$-algebras (inverse limits of $C^{*}$-algebras, also called $L M C^{*}$ algebras) using multipliers, while Van Daele [20] has considered multipliers on Hopf algebras which provide a natural framework to study quantum groups. Therefore, it is important to develop the theory of multipliers for general topological algebras and, in particular, for metrizable topological algebras.

In this paper, we are mainly concerned with the linear topological properties of 
double multiplier algebra $M_{d}(A)$ endowed with the uniform and strong operator topologies $u$ and $s$, respectively. In Section 2, we define multipliers and double multipliers on an algebra $A$ and summarize some basic results for later use. In Section 3, we introduce the $u$ and $s$ topologies on $M_{d}(A)$ and study their properties. Our main results include extensions of some results of Busby [2] and Phillips [14] from $C^{*}$ - and $\sigma-C^{*}$-algebras to more general classes of topological algebras.

2. Preliminaries. Let $A$ be a complex Hausdorff topological algebra in which multiplication is associative and separately continuous. An algebra $A$ is said to be proper (or without order) if $a A=A a=\{0\}$ implies that $a=0$. We note that $A$ is proper in each of the following cases:

(i) $A$ has an identity;

(ii) $A$ is a topological algebra with an approximate identity (e.g., $A$ is a $B^{*}$-algebra);

(iii) $A$ is a topological algebra with an orthogonal basis [5].

For the general theory of topological vector spaces and topological algebras, we refer to the books of Rudin [16] and Mallios [13]. A mapping $T: A \rightarrow A$ is called a multiplier (respectively, left multiplier, right multiplier) on $A$ if $a T(b)=T(a) b$ (respectively $T(a b)=T(a) b, T(a b)=a T(b)$ ) for all $a, b \in A$ (see, e.g., [6]). A pair $(S, T)$ of mappings $S, T: A \rightarrow A$ is called a double multiplier (or a double centralizer) on $A$ if $a S(b)=T(a) b$ for all $a, b \in A$. Let $M(A)$ denote the set of all continuous multipliers on $A$, and let $M_{d}(A)$ denote the set of all double multipliers $(S, T)$ of $A$ with $S$ and $T$ continuous. For convenience, we summarize some basic properties of these multipliers in the following theorems:

THEOREM 2.1. Let $A$ be a proper topological algebra. Then

(a) If $(S, T) \in M_{d}(A)$, then $S$ is a left multiplier and $T$ is a right multiplier on $A$.

(b) Each $T \in M(A)$ is linear; if $(S, T) \in M_{d}(A)$, then $S$ and $T$ are linear.

(c) $M(A)$ is a commutative algebra with composition as multiplication (i.e., $\left(T_{1} T_{2}\right)($ a) $\left.=T_{1}\left(T_{2}(a)\right)\right)$ and it has the identity $I: A \rightarrow A, I(a)=a$.

(d) $M_{d}(A)$ is an algebra with identity $(I, I)$ under the operations

and

$$
\begin{aligned}
(S, T)+\left(S_{1}, T_{1}\right) & =\left(S+S_{1}, T+T_{1}\right), \\
\lambda(S, T) & =(\lambda S, \lambda T),
\end{aligned}
$$

$$
(S, T)\left(S_{1}, T_{1}\right)=\left(S S_{1}, T_{1} T\right), \quad \lambda \in \mathbb{C} .
$$

(e) If $A$ is commutative, then $M_{d}(A)$ is commutative and $M_{d}(A) \equiv M(A)$; in fact, if $(S, T) \in M_{d}(A)$, then $S=T$.

(f) If $A$ is a Banach algebra, then so is $M_{d}(A)$ with the norm given by $\|(S, T)\|=$ $\max \{\|S\|,\|T\|\}$.

Proof. See $[2,7]$.

For any $a \in A$, let $L_{a}, R_{a}: A \rightarrow A$ be given by $L_{a}(x)=a x$ and $R_{a}(x)=x a, x \in A$. Clearly, $\left(L_{a}, R_{a}\right) \in M_{d}(A)$. It is easy to see that, for any $a \in A$ and $(S, T) \in M_{d}(A)$,

$$
\left(L_{a}, R_{a}\right)(S, T)=\left(L_{T(a)}, R_{T(a)}\right), \quad(S, T)\left(L_{a}, R_{a}\right)=\left(L_{S(a)}, R_{S(a)}\right) .
$$


We define a map $\mu: A \rightarrow M_{d}(A)$ by $\mu(a)=\left(L_{a}, R_{a}\right), a \in A$.

TheOREM 2.2. Let A be a Hausdorff topological algebra. Then

(a) $\mu$ is linear, algebra homomorphism, and continuous.

(b) $\mu$ is one-one if and only if $A$ is proper.

(c) $\mu$ is onto if and only if $A$ has identity.

(d) If $A$ is proper, then $\mu(A)$ is a two-sided ideal in $M_{d}(A)$.

Proof. See $[2,7]$.

The following result, due to Johnson [7, 8] (see also Wang [21, p. 1132]), is concerned with the linearity and continuity of multipliers on $A$. For completeness, we include its proof here.

THEOREM 2.3. (a) Suppose that A is a complete metrizable LMC algebra with a uniformly bounded left (right) approximate identity. If $T$ is a left (right) multiplier on $A$, then $T$ is linear and continuous.

(b) Suppose that $A$ is a proper complete metrizable algebra. If $(S, T)$ is a double multiplier on $A$, then $S$ and $T$ are linear and continuous.

Proof. (a) Let $T$ be a left multiplier on $A$. By a generalization of the Cohen's factorization theorem (see [3]), given any sequence $\left\{a_{n}\right\} \subseteq A$ with $a_{n} \rightarrow 0$, there exist $b \in A$ and $\left\{c_{n}\right\} \subseteq A$ with $c_{n} \rightarrow 0$ such that $a_{n}=b c_{n}$ for all $n \geq 1$. To show that $T$ is linear, let $a_{1}, a_{2} \in A$ and $\alpha, \beta \in \mathbb{C}$. Taking $\left\{a_{n}\right\}=\left\{a_{1}, a_{2}, 0,0, \ldots\right\}$, there exist $b, c_{1}, c_{2} \in A$ such that $a_{1}=b c_{1}$ and $a_{2}=b c_{2}$. So,

$$
\begin{aligned}
T\left(\alpha a_{1}+\beta a_{2}\right) & =T\left(b\left(\alpha c_{1}+\beta c_{2}\right)\right)=T(b)\left(\alpha c_{1}+\beta c_{2}\right) \\
& =\alpha T\left(b c_{1}\right)+\beta T\left(b c_{2}\right)=\alpha T\left(a_{1}\right)+\beta T\left(a_{2}\right) .
\end{aligned}
$$

To show that $T$ is continuous, let $\left\{a_{n}\right\} \subseteq A$ with $a_{n} \rightarrow 0$. We can write $a_{n}=b c_{n}$, where $b \in A$ and $\left\{c_{n}\right\} \subseteq A$ with $c_{n} \rightarrow 0$. Hence, $T\left(a_{n}\right)=T\left(b c_{n}\right)=T(b) c_{n} \rightarrow 0$. In the case of a right multiplier, the proof is similar to the above.

(b) Let $(S, T) \in M_{d}(A)$. Then $S$ and $T$ are linear by Theorem 2.1(b). In view of the closed graph theorem [16, Thm. 2.15], it suffices to show that $S$ and $T$ have closed graphs. Let $\left\{a_{\alpha}\right\}$ be a net in $A$ with $a_{\alpha} \rightarrow a \in A$ and $S\left(a_{\alpha}\right) \rightarrow b \in A$. Since multiplication is separately continuous, for any $x \in A, x a_{\alpha} \rightarrow x a$ and $x S\left(a_{\alpha}\right) \rightarrow x b$. Hence,

$$
x S(a)=T(x) a=\lim _{\alpha} T(x) a_{a}=\lim _{\alpha} x S\left(a_{\alpha}\right)=x b .
$$

Since $A$ is proper, we have $S(a)=b$. Hence, $S$ has a closed graph. Similarly, $T$ also has a closed graph.

3. Main results. In the sequel, $A$ denotes a proper Hausdorff topological algebra with multiplication jointly continuous. Following Johnson [7], the uniform operator topology $u$ (respectively, the strong operator topology $s$ ) on $M_{d}(A)$ is defined as the linear topology which has a base of neighborhoods of 0 consisting of all the sets of the form

$$
N(D, W)=\left\{(S, T) \in M_{d}(A): S(D) \subseteq W \text { and } T(D) \subseteq W\right\},
$$


where $D$ is a bounded (respectively, finite) subset of $A$ and $W$ is a neighborhood of 0 in $A$. (The topology $s$ is sometimes called the strict topology; see, e.g., [1, 2, 4, 5, 14, 17, $18,21])$. Clearly, $s \leq u$. It is easy to see that $M_{d}(A)$, endowed with each of $u$ and $s$, is a topological algebra in which multiplication is separately continuous. In [7], Johnson observed that if $A$ is a locally convex barrelled quasi-complete metrizable algebra, then $\left(M_{d}(A), u\right)$ and $\left(M_{d}(A), s\right)$ are quasi-complete. In this section, we consider the completeness and some other properties of $\left(M_{d}(A), u\right)$ and $\left(M_{d}(A), s\right)$ without the local convexity assumption on $A$. We also consider some conditions on $A$ under which $A$ is $u$-closed with respect to the uniform topology (that is, $u$-closed) and dense with respect to the strong operator topology (that is, $s$-dense) in $M_{d}(A)$.

The following result extends [2, Thm. 2.11 and Prop. 3.1] to topological algebras.

THEOREM 3.1. (a) If $A$ is complete and metrizable, then $\left(M_{d}(A), u\right)$ is complete.

(b) If $A$ is complete, then $A$ is a u-closed two-sided ideal in $M_{d}(A)$, under the identification $\mu: a \rightarrow\left(L_{a}, R_{a}\right)$.

Proof. (a) Suppose that $A$ is complete and metrizable and $\left\{\left(S_{\alpha}, T_{\alpha}\right)\right\}$ be a Cauchy net in $\left(M_{d}(A), u\right)$. Then it easily follows that, for each $a \in A,\left\{S_{\alpha}(a)\right\}$ and $\left\{T_{\alpha}(a)\right\}$ are Cauchy nets in $A$. Consequently, the mappings $S, T: A \rightarrow A$, given by $S(a)=\lim _{\alpha} S_{\alpha}(a)$ and $T(a)=\lim _{\alpha} T_{\alpha}(a)(a \in A)$, are well-defined. Further, for any $a, b \in A$,

$$
a S(b)=\lim _{\alpha} a S_{\alpha}(b)=\lim _{\alpha} T_{\alpha}(a) b=T(a) b .
$$

Hence, by Theorem 2.3(b), $(S, T) \in M_{d}(A)$. We now show that $\left(S_{\alpha}, T_{\alpha}\right) \stackrel{u}{\longrightarrow}(S, T)$. Let $D$ be a bounded subset of $A$ and let $W$ be a closed neighborhood of 0 in $A$. There exists an index $\alpha_{0}$ such that

$$
S_{\alpha}(a)-S_{\beta}(a) \in W \quad \text { and } \quad T_{\alpha}(a)-T_{\beta}(a) \in W
$$

for all $a \in D$ and $\alpha, \beta \geq \alpha_{0}$. Since $W$ is closed, fixing $\alpha \geq \alpha_{0}$ and taking $\lim _{\beta}$, we get

$$
S_{\alpha}(a)-S(a) \in W \quad \text { and } \quad T_{\alpha}(a)-T(a) \in W
$$

for all $a \in D$. Hence, for any $\alpha \geq \alpha_{0}$,

$$
\left(S_{\alpha}, T_{\alpha}\right)-(S, T) \in N(D, W) .
$$

Thus, $\left(M_{d}(A), u\right)$ is complete.

(b) Suppose that $A$ is complete. We have already seen in Theorem 2.2 that $\mu(A)$ is a two-sided ideal in $M_{d}(A)$. To show that $\mu(A)$ is $u$-closed in $M_{d}(A)$, let $(S, T) \in M_{d}(A)$ with $(S, T) \in \overline{\mu(A)}^{u}$. There exists a net $\left\{a_{\alpha} \subseteq A\right\}$ such that $\left(L_{a_{\alpha}}, R_{a_{\alpha}}\right) \stackrel{u}{\longrightarrow}(S, T)$. Then, for any $b \in A, a_{\alpha} b \rightarrow S(b)$ and $b a_{\alpha} \rightarrow T(b)$, and so $\left\{a_{\alpha} b\right\}$ and $\left\{b a_{\alpha}\right\}$ are Cauchy nets in $A$. Since $A$ is proper, it follows easily that $\left\{a_{\alpha}\right\}$ is a Cauchy net in $A$. Since $A$ is complete, $a_{\alpha} \rightarrow a \in A$. Hence, by continuity of $\mu$,

$$
(S, T)=\lim _{\alpha} \mu\left(a_{\alpha}\right)=\mu(a)=\left(L_{a}, R_{a}\right),
$$

and so $(S, T) \in \mu(A)$. Thus, $\mu(A)$ is $u$-closed in $M_{d}(A)$. 
The following result was proved in [2] for $A$, a $C^{*}$-algebra and in [14] for $A$, a pro$C^{*}$-algebra.

THEOREM 3.2. (a) If $A$ is complete and metrizable, then $\left(M_{d}(A), s\right)$ is complete.

(b) If $A$ is complete and has a two-sided approximate identity (not necessarily bounded), then $A$ is $s$-dense in $M_{d}(A)$.

Proof. (a) Suppose that $A$ is complete and metrizable, and let $\left\{\left(S_{\alpha}, T_{\alpha}\right)\right\}$ be a Cauchy net in $\left(M_{d}(A), s\right)$. Then, for any $a \in A,\left\{S_{\alpha}(a)\right\}$ and $\left\{T_{\alpha}(a)\right\}$ are Cauchy nets in $A$. Consequently, the mappings $S, T: A \rightarrow A$ given by $S(a)=\lim _{\alpha} S_{\alpha}(a)$ and $T(a)=\lim _{\alpha} T_{\alpha}(a)(a \in A)$ are well-defined and linear. Further, for any $a, b \in A$,

$$
a S(b)=\lim _{\alpha} a S_{\alpha}(b)=\lim _{\alpha} T_{\alpha}(a) b=T(a) b,
$$

and so by Theorem $2.3(\mathrm{~b}),(S, T) \in M_{d}(A)$. To show that $\left(S_{\alpha}, T_{\alpha}\right) \stackrel{s}{\rightarrow}(S, T)$, let $D$ be a finite subset of $A$ and let $W$ be a neighborhood of 0 in $A$. There exists an index $\alpha_{0}$ such that

$$
S_{\alpha}(a)-S(a) \in W \quad \text { and } \quad T_{\alpha}(a)-T(a) \in W
$$

for all $\alpha \geq \alpha_{0}$ and all $a \in D$ (since $D$ is finite). Hence, $\left(S_{\alpha}, T_{\alpha}\right)-(S, T) \in N(D, W)$ for all $\alpha \geq \alpha_{0}$. Thus, $\left(M_{d}(A), s\right)$ is complete.

(b) Suppose that $A$ is complete, and let $\left\{e_{\lambda}: \lambda \in I\right\}$ be a two-sided approximate identity for $A$. We need to show that $\mu(A)$ is $s$-dense in $M_{d}(A)$. Let $(S, T) \in M_{d}(A)$, and let $D$ be a finite subset of $A$ and $W$ a neighborhood of 0 in $A$. We claim that, for some $\lambda \in I, \mu\left(T\left(e_{\lambda}\right)\right)-(S, T) \in N(D, W)$. Now, by definition, $e_{\lambda} b \rightarrow b$ and $b e_{\lambda} \rightarrow b$ for all $b \in A$. Since $D$ is finite, we can choose a $\lambda_{0} \in I$ such that

$$
e_{\lambda} S(a)-S(a) \in W \quad \text { and } \quad T\left(a e_{\lambda}\right)-T(a) \in W
$$

for all $a \in D$ and all $\lambda \geq \lambda_{0}$. Then, for any $a \in D$ and $\lambda \geq \lambda_{0}$,

$$
L_{T\left(e_{\lambda}\right)}(a)-S(a)=T\left(e_{\lambda}\right) a-S(a)=e_{\lambda} S(a)-S(a) \in W
$$

and

$$
L_{T\left(e_{\lambda}\right)}(a)-T(a)=a T\left(e_{\lambda}\right)-T(a)=T\left(a e_{\lambda}\right)-T(a) \in W .
$$

Thus, $\mu(A)$ is $s$-dense in $M_{d}(A)$ and this completes the proof.

As an application, we get an extension of Tomiuk's result [18, Lem. 2.1].

COROLLARY 3.3. Suppose that A is complete and has two-sided approximate identity, and let $J$ be a two-sided ideal in $A$. Then $J$ is u-dense in $A$ if and only if $J$ is s-dense in $M_{d}(A)$.

Proof. Suppose that $\bar{J}^{u}=A$. We need to show that $\overline{\mu(J)}^{s}=M_{d}(A)$. Since $s \leq u$; $\bar{J}^{u} \subseteq \bar{J}^{s}$; hence $A \subseteq \bar{J}^{s}$. By Theorem 3.2(b), $\overline{\mu(A)}^{s}=M_{d}(A)$, and so $M_{d}(A) \subseteq \overline{\mu(J)}^{s}$. Conversely, suppose that $\overline{\mu(J)} s=M_{d}(A)$. Let $\left\{e_{\lambda}: \lambda \in I\right\}$ be a two-sided approximate identity for $A$, and let $a \in A$. Since $\mu(A) \subseteq M_{d}(A)=\overline{\mu(J)}^{s}, \mu(a) \in \overline{\mu(J)}^{s}$ and so there 
exists a net $\left\{a_{\alpha}\right\} \subseteq J$ such that $\mu\left(a_{\alpha}\right) \stackrel{s}{\longrightarrow} \mu(a)$. Then, for each $\lambda \in I, \lim _{\alpha} \mu\left(a_{\alpha} e_{\lambda}\right)-$ $\mu\left(a e_{\lambda}\right)=0$. Now, $a_{\alpha} e_{\lambda} \in J$ and so $\mu\left(a_{\alpha} e_{\lambda}\right) \in \overline{\mu(J)}^{s}$ for each $\lambda \in I$. Consequently, $a \in \bar{J}^{u}$, which shows that $\bar{J}^{u}=A$.

A topological vector space (TVS) $E$ is called ultrabornological if every bounded linear map from $E$ into any TVS is continuous. It follows from [16, Thm. 1.32] that every metrizable TVS is ultrabornological.

THEOREM 3.4. Suppose that $A$ is complete and metrizable. Then

(a) $s$ and $u$ have the same bounded sets.

(b) If $\left(M_{d}(A), s\right)$ is ultrabornological, then $s=u$ on $M_{d}(A)$.

Proof. (a) Since $s \leq u$, every $u$-bounded set in $M_{d}(A)$ is bounded. Conversely, let $H$ be any $s$-bounded set in $M_{d}(A)$. Then, if $a \in A$, for each neighborhood $V$ of 0 in $A$, there exists a constant $r_{a}>0$ such that $H \subseteq r_{a} N(\{a\}, V)$. This implies that, for each $a \in A,\{(S(a), T(a)):(S, T) \in H\}$ is a bounded set in $A \times A$. By the principle of uniform boundedness [16, Thm. 2.5], the collection $\{S, T:(S, T) \in H\}$ is equicontinuous. Then it follows from [16, Thm. 2.4] that, for any bounded set $D$ in $A$ and a neighborhood $W$ of 0 in $A$, there exists a constant $r>0$ such that $H \subseteq r N(D, W)$. Thus, $H$ is $u$-bounded.

(b) By (a), the identity map $i:\left(M_{d}(A), s\right) \rightarrow\left(M_{d}(A), u\right)$ is bounded. So, by hypothesis, $i$ is continuous and hence $u \leq s$.

Now, let $U$ be a proper topological algebra which contains $A$ as a closed two-sided ideal. For any $y \in U$, define $L_{y}, R_{y}: A \rightarrow A$ by $L_{y}(a)=y a$ and $R_{y}(a)=a y, a \in A$. Clearly, $\left(L_{y}, R_{y}\right) \in M_{d}(A)$. Define a map $\mu^{\prime}=U \rightarrow M_{d}(A)$ by $\mu^{\prime}(y)=\left(L_{y}, R_{y}\right), y \in U$. Then, as in [2, Prop. 3.7], $\mu^{\prime}$ is a unique homomorphism satisfying $\mu^{\prime}(a)=\mu(a)$ for all $a \in A$; further, $\operatorname{Ker} \mu^{\prime}=\{y \in U: y A=\{0\}\}$. We next define a topology $s^{\prime}$ on $U$ as the linear topology which has a base of neighborhoods of 0 in $U$ consisting of all the sets of the form

$$
N(D, W)=\{y \in U: y D \subseteq W, D y \subseteq W\},
$$

where $D$ is a finite subset of $A$ and $W$ is a neighborhood of 0 in $A$. If $u^{\prime}$ is the given topology on $U$, then, clearly, $s^{\prime} \leq u^{\prime}$.

THEOREM 3.5. Let $U, A, \mu^{\prime}$, and $s^{\prime}$ be as above. Then

(a) $\mu^{\prime}:\left(U, s^{\prime}\right) \rightarrow\left(M_{d}(A), s\right)$ is continuous and open onto $\mu^{\prime}(U)$.

(b) Suppose that $A$ is complete and metrizable, and has two-sided approximate identity. If $\left(U, s^{\prime}\right)$ is complete and $\operatorname{Ker} \mu^{\prime}=\{0\}$, then $\mu^{\prime}(U)=M_{d}(A)$.

Proof. (a) Let $\left\{y_{\alpha}\right\}$ be a net in $U$ with $y_{\alpha} \stackrel{s^{\prime}}{\rightarrow} y \in U$. This means, for each $a \in A$, $y_{\alpha} a \rightarrow y a$ and $a y_{\alpha} \rightarrow a y$ in $A$, that is $\left(L_{y_{\alpha}}, R_{y_{\alpha}}\right) \stackrel{s^{\prime}}{\rightarrow}\left(L_{y}, R_{y}\right)$ in $M_{d}(A)$. Thus, $\mu^{\prime}$ is continuous and open onto $\mu^{\prime}(U)$.

(b) It follows from the hypothesis that $\mu^{\prime}(U)$ is complete and hence a closed subset of $\left(M_{d}(A), s\right)$. Since $A \subseteq U$ and $\mu^{\prime}(A)$ is $s$-dense in $M_{d}(A)$ (Theorem 3.2(b)), $\mu^{\prime}(U)$ is $s$-dense in $M_{d}(A)$. Thus, $\mu^{\prime}(U)=M_{d}(A)$.

In the following paragraph, we denote the first and the second component of a double multiplier $T$ on an algebra by $[T]^{\prime}$ and $[T]^{\prime \prime}$, respectively. Let $A$ and $B$ be two proper Hausdorff topological algebras, and let $\phi: A \rightarrow B$ be an onto homomorphism. 
Then by [7, Thm. 4] and [2, Prop. 3.8], there exists a unique (extension) homomorphism map $\tilde{\phi}: M_{d}(A) \rightarrow M_{d}(B)$ given by

$$
[\tilde{\phi}(T)]^{\prime}(\phi(a))=\phi\left([T]^{\prime}(a)\right), \quad[\tilde{\phi}(T)]^{\prime \prime}(\phi(a))=\phi\left([T]^{\prime \prime}(a)\right)
$$

for $T \in M_{d}(A)$ and $a \in A$.

THEOREM 3.6. Let $A, B, \phi$, and $\tilde{\phi}$ be as above, and let $s_{A}$ and $s_{B}$ denote the strong operator topologies on $M_{d}(A)$ and $M_{d}(B)$, respectively. If $\phi: A \rightarrow B$ is continuous, then so is the map $\tilde{\phi}:\left(M_{d}(A), s_{A}\right) \rightarrow\left(M_{d}(B), s_{B}\right)$.

Proof. Suppose that $\phi: A \rightarrow B$ is continuous, and let $\left\{T_{\alpha}\right\}$ be a net in $M_{d}(A)$ with $T_{\alpha} \stackrel{S_{A}}{\longrightarrow} T \in M_{d}(A)$. Then, if $b \in B$ and $b=\phi(a)$ for $a \in A$,

$$
\left[\tilde{\phi}\left(T_{\alpha}\right)\right]^{\prime}(b)=\phi\left(\left[T_{\alpha}\right]^{\prime}(a)\right) \longrightarrow \phi\left([T]^{\prime}(a)\right)=[\tilde{\phi}(T)]^{\prime}(b),
$$

and similarly $\left[\tilde{\phi}\left(T_{\alpha}\right)\right]^{\prime \prime}(b) \rightarrow[\tilde{\phi}(T)]^{\prime \prime}(b)$ in $B$. Thus, $\tilde{\phi}\left(T_{\alpha}\right) \stackrel{S_{B}}{\longrightarrow} \tilde{\phi}(T)$, showing that $\tilde{\phi}$ is continuous.

Acknowledgement. One of the authors (A. B. Thaheem) wishes to acknowledge the support provided by King Fahd University of Petroleum and Minerals during this research.

\section{REFERENCES}

[1] Z. Argün and K. Rowlands, On quasi-multipliers, Studia Math. 108 (1994), no. 3, 217-245. MR 95f:46078. Zbl 824.46053.

[2] R. C. Busby, Double centralizers and extensions of $C^{*}$-algebras, Trans. Amer. Math. Soc. 132 (1968), 79-99. MR 37\#770. Zbl 165.15501.

[3] I. G. Craw, Factorisation in Frechét algebras, J. London Math. Soc. 44 (1969), 607-611. MR 39\#3311. Zbl 175.14204.

[4] J. W. Davenport, The strict dual of $B^{*}$-algebras, Proc. Amer. Math. Soc. 65 (1977), no. 2, 309-312. MR 58 30285. Zbl 365.46047.

[5] R. A. Fontenot, The double centralizer algebra as a linear space, Proc. Amer. Math. Soc. 53 (1975), no. 1, 99-103. MR 53 11385. Zbl 315.46049.

[6] T. Husain, Multipliers of topological algebras, Dissertationes Math. (Rozprawy Mat.) 285 (1989), 40. MR 90h:46079. Zbl 676.46038.

[7] B. E. Johnson, An introduction to the theory of centralizers, Proc. London Math. Soc. (3) 14 (1964), 299-320. MR 28\#2450. Zbl 143.36102.

[8] _ Continuity of centralisers on Banach algebras, J. London Math. Soc. 41 (1966), 639-640. MR 34\#629. Zbl 143.36103.

[9] R. Larsen, An Introduction to the Theory of Multipliers, Die Grundlehren der mathematischen Wissenschaften, vol. 175, Springer-Verlag, New York, Heidelberg, 1971. MR 55 8695. Zbl 213.13301.

[10] G. Lassner, Topological algebras of operators, Rep. Math. Phys. 3 (1972), no. 4, 279-293. MR 48889.

[11]__ Topological algebras and their applications in quantum statistics, Wiss. Z. Karl Marx Univ. Leipzig Math. Natur. Reihe 30 (1981), no. 6, 572-595. MR 83d:82011. Zbl 483.47027.

[12] A. J. Lazar and D. C. Taylor, Multipliers of Pedersen's ideal, Mem. Amer. Math. Soc. 5 (1976), no. 169, 111. MR 54 944. Zbl 322.46063.

[13] A. Mallios, Topological Algebras. Selected Topics, North-Holland Mathematics Studies, vol. 124, North-Holland Publishing Co., Amsterdam, New York, 1986, Mathematical Notes, 109. MR 87m:46099. Zbl 597.46046. 
[14] N. C. Phillips, Inverse limits of $C^{*}$-algebras, J. Operator Theory 19 (1988), no. 1, 159-195. MR 90c:46090. Zbl 662.46063.

[15] _ Inner derivations on $\sigma$-C $C^{*}$-algebras, Math. Nachr. 176 (1995), 243-247. MR 96j:46069. Zbl 838.46055.

[16] W. Rudin, Functional Analysis, McGraw-Hill Series in Higher Mathematics, McGraw-Hill Book Co., New York, Dusseldorf, Johannesburg, 1973. MR 51 1315. Zbl 253.46001.

[17] D. C. Taylor, The strict topology for double centralizer algebras, Trans. Amer. Math. Soc. 150 (1970), 633-643. MR 44\#7302. Zbl 204.14701.

[18] B. J. Tomiuk, Multipliers on Banach algebras, Studia Math. 54 (1975/1976), no. 3, 267-283. MR 53 6328. Zbl 319.46033.

[19] B. J. Tomiuk and P. K. Wong, The Arens product and duality in $B^{*}$-algebras, Proc. Amer. Math. Soc. 25 (1970), 529-535. MR 41\#4256. Zbl 198.17902.

[20] A. van Daele, Multiplier Hopf algebras, Trans. Amer. Math. Soc. 342 (1994), no. 2, 917-932. MR 94h:16075. Zbl 809.16047.

[21] J. K. Wang, Multipliers of commutative Banach algebras, Pacific J. Math. 11 (1961), 11311149. MR 25\#1462. Zbl 127.33302.

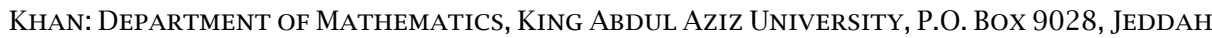
21413, SAUDI ARABIA

MOHAMMAD: DEPARTMENT OF MATHEMATICS, QUAID-I-AZAM UNIVERSITY, ISLAMABAD-45320, PAKISTAN

THAHEEM: DEPARTMENT OF MATHEMATICAL SCIENCES, King FAHD UNIVERSITY OF PETROLEUM AND MinERALS, DHAHRAN 31261, SAUDI ARABIA

E-mail address: athaheem@kfupm.edu.sa 


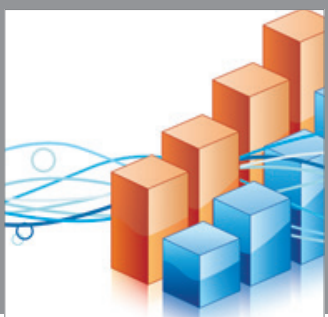

Advances in

Operations Research

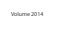

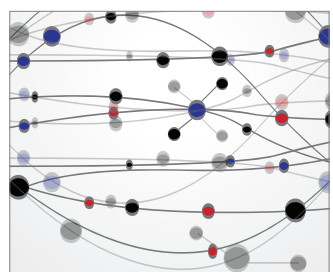

\section{The Scientific} World Journal
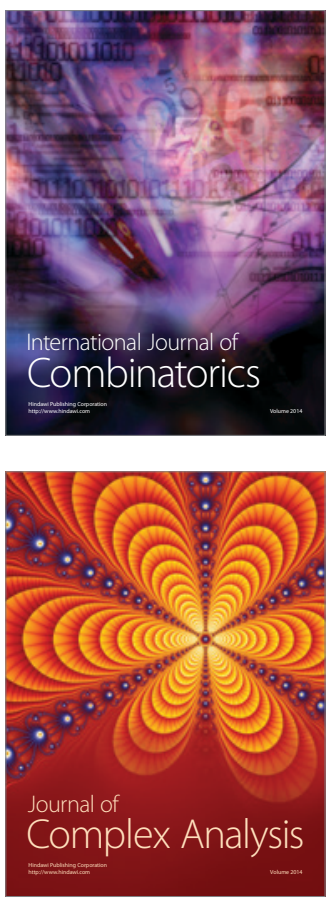

International Journal of

Mathematics and

Mathematical

Sciences
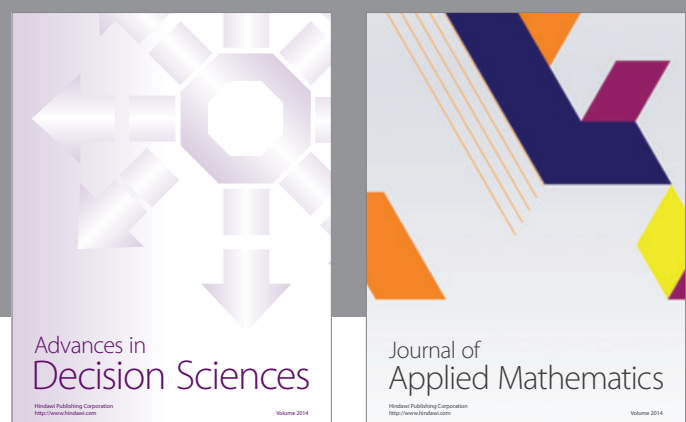

Journal of

Applied Mathematics
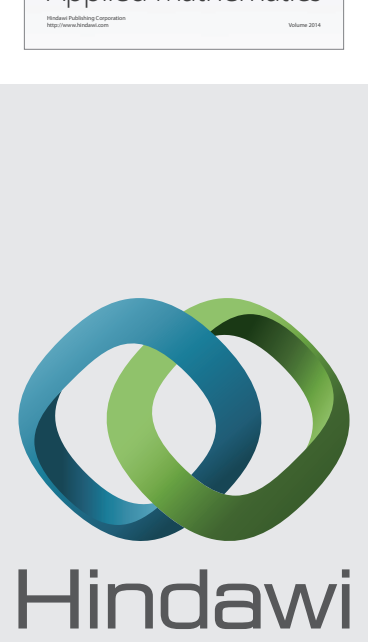

Submit your manuscripts at http://www.hindawi.com
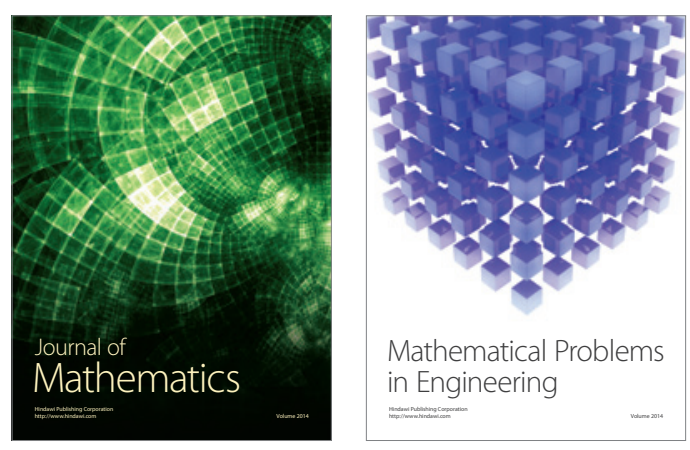

Mathematical Problems in Engineering
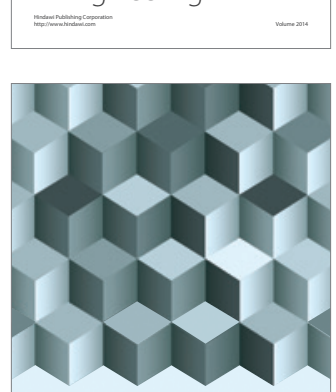

Journal of

Function Spaces
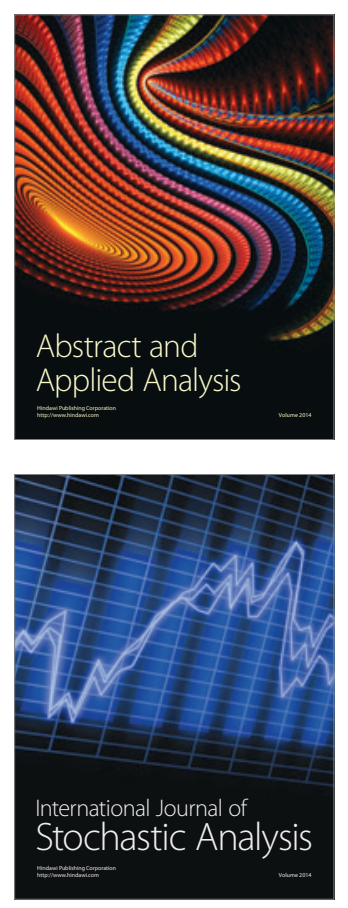

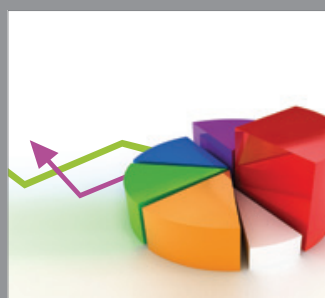

ournal of

Probability and Statistics

Promensencen
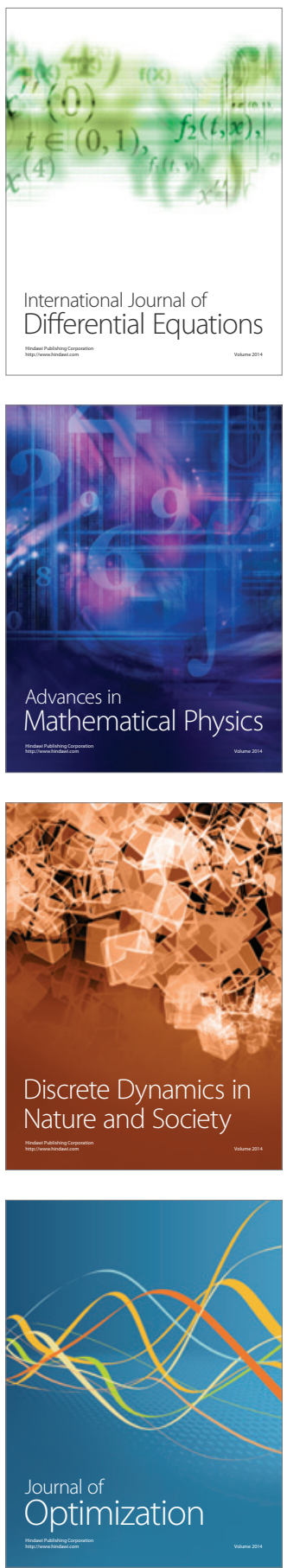